\title{
Multiphoton Mass Spectrometry: Metastables
}

\author{
H. KÜHLEWIND, U. BOESL, R. WEINKAUF, H. J. NEUSSER \\ and E. W. SCHLAG
}

Institut für Physikalische und Theoretische Chemie, Technische Universität München, Lichtenbergstraße 4, D-8046 Garching, Germany

Multiphoton absorption in molecules is a facile method of producing ions. This technique enables one to use visible or near ultraviolet light for the production of molecular ions. This ionization is shown to proceed with near $100 \%$ efficiency in a well localized source, the focus of the laser, and a well defined time, the excitation pulse length of the laser. This makes for an ideal ion source for a mass spectrometer. A time-of-flight configuration will be discussed with a present mass resolution of 3000-4000. This high transmission TOF enables a complete analysis of all ions from a single laser shot, providing suitable electronics is available. This unit can also be used to obtain new information on the behavior of ion decay processes near their energetic thresholds (metastables). The method provides detailed information when the laser wavelength or its intensity is tuned. Comparison to conventional mass spectrometry will be given.

\section{INTRODUCTION}

By means of the intense light of pulsed lasers it is possible that a single atom or molecule absorbs several photons from the radiation field to be excited to the ionization continuum. This multi-photon ionization (MPI) is a new versatile method for the production of polyatomic molecular ions in a mass spectrometer. ${ }^{1-8} \mathrm{~A}$ very interesting advantage of this photoionization method is that visible or near ultraviolet light may be used for ionization and the complex vacuum UV technique is not required. Employing laser light of variable wavelength Boesl $e t$ al. ${ }^{1}$ as well as Zandee et al. ${ }^{9}$ pointed out some of the complementary characteristics of multi-photon mass spectrometry. Boesl et al. ${ }^{10}$ showed that this ionization method is highly wavelength selective due to the first absorption step and therefore the isotopic species ${ }^{13} \mathrm{CC}_{5} \mathrm{H}_{6}^{+}$can be preferentially ionized in a natural 
isotopic mixture of benzene shifting the wavelength by $1.6 \mathrm{~cm}^{-1}$ to the blue from the $6_{0}^{1}$ absorption band of the $S_{1} \leftarrow S_{0}$ transition of light benzene. In their early work ${ }^{1}$ they showed that the laser intensity can be adjusted so that the parent ion peak $\mathrm{C}_{6} \mathrm{H}_{6}^{+}$appears exclusively (soft ionization without fragmentation); in conventional electron impact mass spectrometry this exclusive production of parent ions is difficult or inefficient. Thus MPI is a good method for the determination of molecular weights via "soft" ionization. On the other hand, Zandee $e t a l .^{2}$ demonstrated that with increasing intensity of the laser light a large number of photons (typically 5-15) are absorbed during the laser pulse, thus producing fragmentation of the parent ions with increasing intensity. This intensity dependence of the fragmentation pattern of a polyatomic molecule adds a third dimension to this new kind of mass spectrometry, which is already two-dimensional in mass and wavelength.

\section{GENERAL DESCRIPTION OF THE MPI PROCESS-MECHANISM}

For the observation of a two-photon ionization with UV light of some $10^{6} \mathrm{~W} / \mathrm{cm}^{2}$ the first absorption step must involve a transition into a real intermediate level of the $S_{1}$-system. The second photon then transports the molecule into the ionization continuum. As a consequence the ion current as function of the wavelength is modulated by the spectral properties of the $S_{1}$-state as well as by the final state above the ionization potential. Sharp spectral features are, however, only expected for the $S_{1} \leftarrow S_{0}$ transition, whereas the final state above the ionization potential is generally less structured.

In the case of high intensity, additional photons are absorbed after having crossed the ionization potential. The absorption could either proceed via the neutral molecule (Figure 1a) or the parent ion (Figure $1 \mathrm{~b}, \mathrm{c})$. In the latter case ionization is proposed to occur at the earliest possible energy level, i.e., after the first photon has crossed the ionization threshold. The absorption then switches from the neutral molecule to the parent ion and at higher energy levels from the parent ions to fragment ions etc. (Figure 1b). This mechanism is termed ladder switching. ${ }^{11}$ The climbing in the energy ladder to ionizing or autoionizing states of the neutrals has been referred to as the autoionization tree model. A model intermediate between these two extremal 


\section{AUTOIONIZATION TREE}

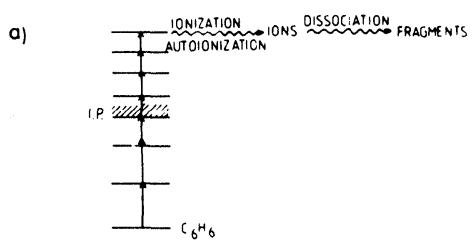

LADDER SWITCHING

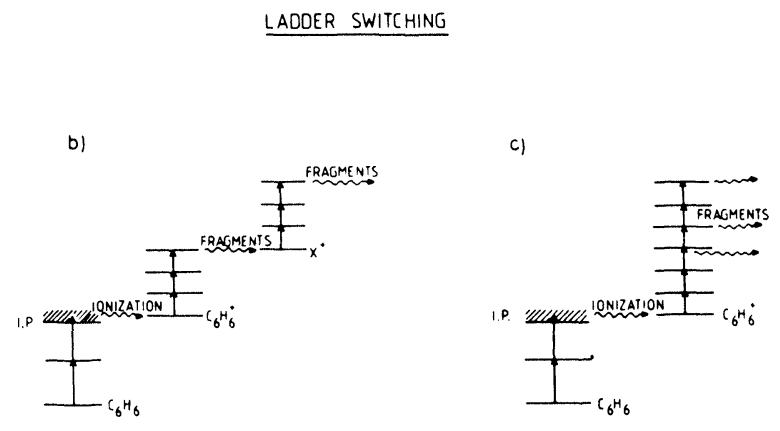

FIGURE 1 Several models for UV multi-photon ionization and fragmentation of a polyatomic molecule (e.g., $\mathrm{C}_{6} \mathrm{H}_{6}$ ).

mechanisms would involve the absorption of additional photons in the molecular ions and no switching from the parent ion to the fragment ion ${ }^{12}$ (Figure 1c).

Theoretical considerations and detailed kinetic calculations ${ }^{13}$ suggest that the ladder switching is typical for most molecular systems for which a RRKM mechanism, i.e., statistical redistribution of energy among all degrees of freedom can be presumed. After the absorption of two or three additional photons the energy of the molecular ions is so large that they will dissociate rapidly into fragment ions during the laser pulse. These fragments will again absorb two or three photons, leading again to rapid dissociation. These fragmentation processes under consideration are sufficiently rapid that several ladder switches can occur during a laser pulse of typical 5-10 ns. Boesl et $a l .{ }^{11,14}$ carried out a series of experiments in which they first experimentally proved this ladder switching mechanism for the case of benzene. In a two color experiment and a further experiment with two colors with the second color separated not only in time but also 
in space ${ }^{14}$ they showed that photon absorption indeed switches from the neutral benzene to the molecular ion and that the parent ion as well as fragment ions are indeed able to absorb further photons to produce fragments of smaller mass. Further experimental results corroborating the ladder switching mechanism will be presented in this paper in the section on metastable ions.

\section{MASS SPECTROMETER AND EXPERIMENTAL SET UP}

In a MPI process ions are produced in a very tight focus of $c a .100 \mu \mathrm{m}$ diameter as well as within a very short time interval of typically $5-10 \mathrm{~ns}$. Therefore this ionization method is an ideal tool for the separation of ions of different masses by the time-of-flight (TOF) technique. Here all ions are accelerated into a field free drift tube, where they have a kinetic energy of $e U_{\text {acc }}$, where $e$ is the elementary charge and $U_{\text {acc }}$ the acceleration voltage. They are separated in time by their different velocity due to their different mass $m$. Such TOF instruments are time-honored instruments in mass spectrometry but their benefit is limited by poor space and time resolution. The important advantage of the TOF technique is the near unity transmission for ions and the fact that all ions originating from a single laser pulse are recorded in the spectrum since there are no ion selecting parts in the instrument; in conventional scanning instruments only ions of one mass reach the detector, the remaining ions being discarded. These two characteristics of the TOF technique and the good space and time definition of MPI make the combination of these two techniques to an ideal mass spectrometric method for ion studies and trace analysis. In our recent experiments we used a less conventional, new technique of TOF mass analysis, the reflectron TOF spectrometer. ${ }^{15,16,18}$ A scheme of the reflectron TOF mass spectrometer is shown in Figure 2. The configuration of the reflecting field allows for the compensation of energy differences of the ions due to a diffuse ion source, the natural thermal kinetic energy, the Coulomb energy and kinetic energy release of fragmentation processes. The ions are produced in an effusive molecular beam in front of a nozzle by resonantly enhanced two-photon ionization with the frequencydoubled output of a nitrogen laser pumped dye laser and drawn into a drift region of $82 \mathrm{~cm}$ length. After having passed this tube they are 


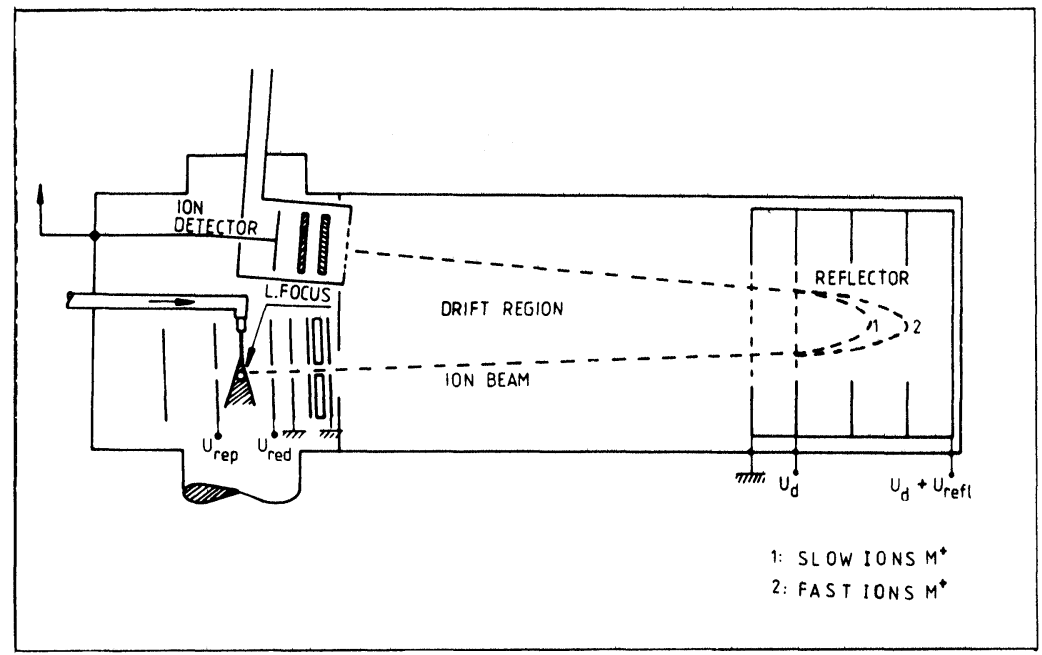

FIGURE 2 Scheme of the reflecting field time-of-flight instrument (reflectron). It is illustrated how kinetic energy differences of the same ion species $M^{+}$are compensated in the reflecting field due to the different flight trajectories.

rapidly decelerated in a variable deceleration field $U_{d}$ and enter the soft reflecting field. Due to a residence time in the reflecting field of about $10 \mu \mathrm{s}$ differences in time-of-flight for a particular ion species are compensated, since faster ions more deeply penetrate into the reflecting field than slower ions of the same mass but different kinetic energy. Therefore they remain there for a longer time than ions with lower kinetic energy. For an optimal set of voltages the half width of the benzene ion mass peak in this way can be reduced from about $200 \mathrm{~ns}$ to only $8 \mathrm{~ns}$ (Figure 3 ). With a difference in time-of-flight for two neighboring mass peaks of $400 \mathrm{~ns}$ we obtained a mass resolution $(M / \Delta M)$ of 3900 at $78 \mathrm{amu} .{ }^{16}$ It is conceivable that this mass resolution could be improved to the 10000 range. In our experiments two microchannel plates Varian VUW-8992 in series are used as ion detector in order to shorten the recovery time of the plates. The signal of the channel plates is impedance matched and then digitized in a transient digitizer (Tektronix 7912). For weak signals averaging over several laser shots is possible with the wave processing system WP 2221 (Tektronix). 


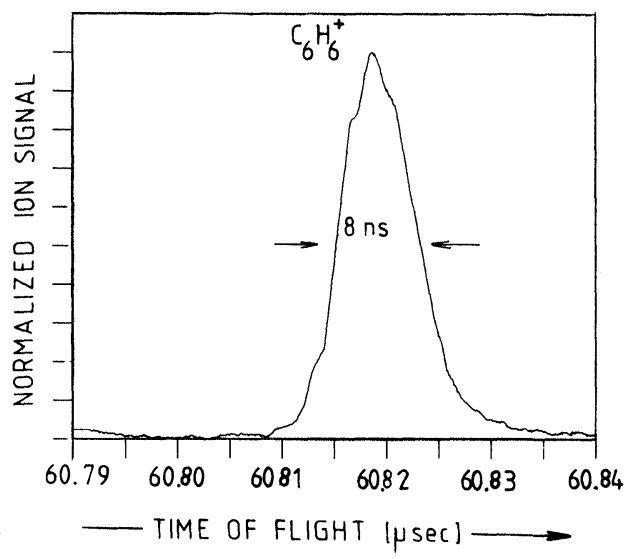

FIGURE 3 Signal of the benzene ions $\mathrm{C}_{6} \mathrm{H}_{6}^{+}$in the energy corrected MPI time-offlight mass spectrum of benzene. Note the half width of only $8 \mathrm{~ns}$.

\section{METASTABLE FRAGMENTATION CHANNELS}

One of the classic problems in mass spectrometry is the investigation of metastable ions and the measurement of decay rate constants as function of internal ion energy. Metastables as such are seen in multi-photon mass spectrometry much as in electron impact mass spectrometry. ${ }^{17}$ Fragment ions produced in the ionization region within $10 \mathrm{~ns}$ are correctly mass detected in a TOF mass spectrometer. Slower dissociation processes may occur in the acceleration region at a time scale of about $1 \mu \mathrm{sec}$ or in the field free drift region on a time scale of about $10 \mu \mathrm{s}$. These two groups of ions are entitled metastable ions. In a conventional TOF instrument dissociation in the acceleration region produces an asymmetric broadening of the fragment mass peaks since the place of dissociation within the accelerating field and hence the kinetic energy of the fragments is not exactly defined. Fragment ions produced in the field free drift region have the same velocity as their precursor ions according to their kinetic energy $E_{\text {kin }}=e U_{\text {acc }} \times m / M$, when $m$ is the fragment ion mass and $M$ the precursor ion mass. Hence these fragments ions $m^{+}$reach the detector after the time-of-flight of the parent ions $\mathrm{M}^{+}$and therefore cannot be observed. If at least partial energy correction is obtained within the reflecting field the fragment ion signal is separated from the precursor 
ion signal. Therefore the fragments produced in the drift tube on a time scale of about $10 \mu \mathrm{s}$ are observable in the time-of-flight spectrum as sharp signals. This greatly extends the decay time range of metastable processes to be observed.

In the case of large energy deviations, i.e., large mass changes during the metastable decay, the energy deviation is only partially corrected and the metastable fragments $m^{+}$reach the detector later than the fragments $\mathrm{m}^{+}$produced in the ionization region but earlier than their precursors (Figure 4a). As example for this case the elimination of acetylene $\mathrm{C}_{2} \mathrm{H}_{2}$ and $\mathrm{C}_{3} \mathrm{H}_{3}$ from the benzene cation $\mathrm{C}_{6} \mathrm{H}_{6}^{+}$is given (Eq. 1). ${ }^{18}$

$$
\begin{aligned}
& \mathrm{C}_{6} \mathrm{H}_{6}^{+} \longrightarrow \mathrm{C}_{4} \mathrm{H}_{4}^{+}+\mathrm{C}_{2} \mathrm{H}_{2} \\
& \mathrm{C}_{6} \mathrm{H}_{6}^{+} \longrightarrow \mathrm{C}_{3} \mathrm{H}_{3}^{+}+\mathrm{C}_{3} \mathrm{H}_{3}
\end{aligned}
$$

Signals of fragments $\mathrm{m}^{+}$produced by a metastable decay in the acceleration region are observed as an asymmetric wing of the ion signal $\mathrm{m}^{+}$produced by ion fragmentation in the laser focus. This wing covers the mass range from that ion to the ions $\mathrm{m}^{+}$produced in the drift tube. A scheme of the corresponding uncorrected spectrum is shown in Figure 4b. Note the much more broadened wing and the overlap of the fragments $m^{+}$produced in the drift region and the precursors. On the other hand for small energy deviations, i.e., small mass changes during the fragmentation process this energy deviation can be even completely corrected by the reflector set up. Then the metastable fragment ions arrive at the detector simultaneously with those fragments produced already in the ion source (Figure 4c). As example the $\mathrm{H}$-loss channels of benzene $\mathrm{C}_{6} \mathrm{H}_{6}^{+}$(Eq. 2 and Eq. 3 ) are given. ${ }^{16}$

$$
\begin{aligned}
& \mathrm{C}_{6} \mathrm{H}_{6}^{+} \longrightarrow \mathrm{C}_{6} \mathrm{H}_{5}^{+}+\mathrm{H} \\
& \mathrm{C}_{6} \mathrm{H}_{6}^{+} \longrightarrow \mathrm{C}_{6} \mathrm{H}_{4}^{+}+\mathrm{H}_{2}
\end{aligned}
$$

In Figure $5 \mathrm{a}$ the signal of the ions $\mathrm{C}_{6} \mathrm{H}_{6}^{+}$and the metastable fragments $\mathrm{C}_{6} \mathrm{H}_{5}^{+}$and $\mathrm{C}_{6} \mathrm{H}_{4}^{+}$in the corrected spectrum is shown. The excitation wavelength here is $2590.1 \mathrm{~A}$. Note the amount of fragments $\mathrm{C}_{6} \mathrm{H}_{5}^{+}$ and $\mathrm{C}_{6} \mathrm{H}_{4}^{+}$produced in the drift region for this photon energy. In the uncorrected spectrum (Figure $5 \mathrm{~b}$ ) these fragments are absent, since the corresponding signal overlaps with the parent ion signal. The 

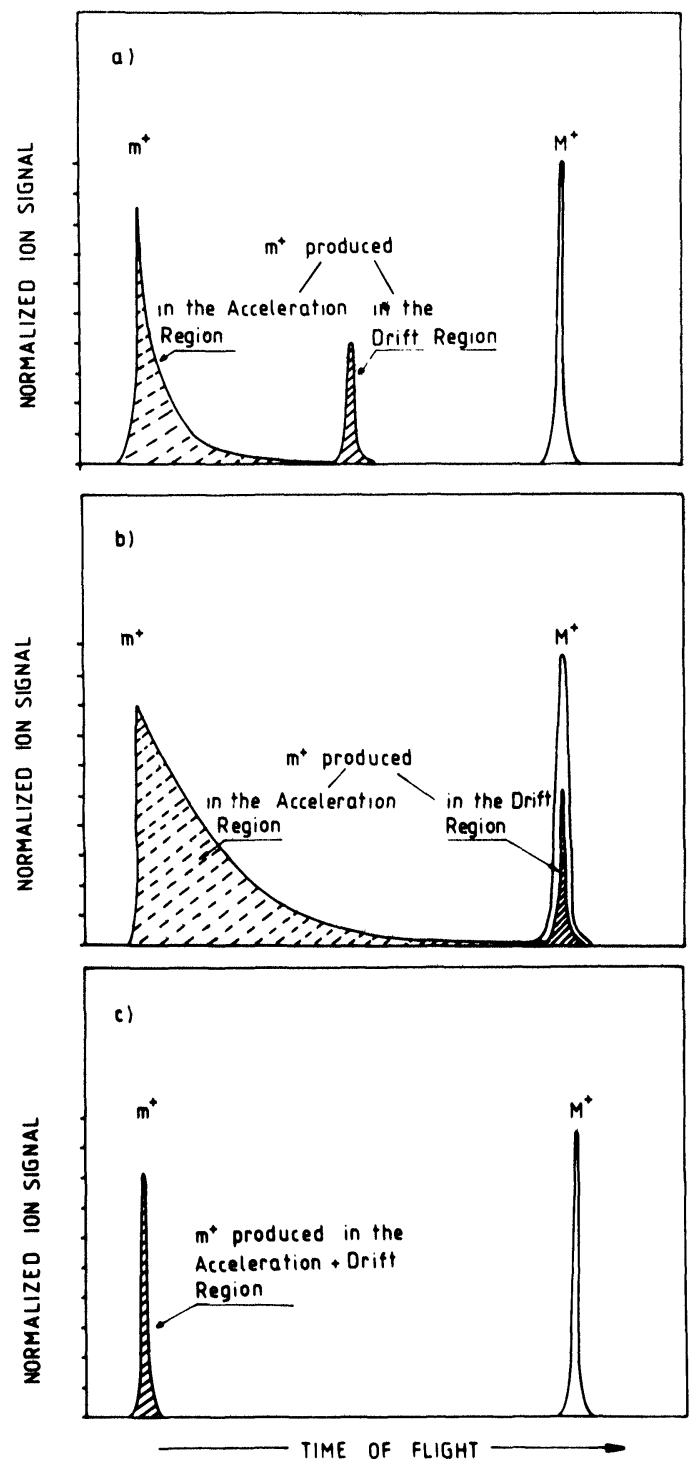

FIGURE 4 Schematic mass spectra of fragments $\mathrm{m}^{+}$produced by a metastable decay of parent ions $\mathrm{M}^{+}$in the acceleration and drift region of the reflectron time-of-flight mass spectrometer. (a) The energy shift of $\mathrm{m}^{+}$due to the metastable decay is partially corrected with the reflecting field. (b) The energy shift of $\mathrm{m}^{+}$due to the metastable decay is not corrected. (c) The energy shift of $\mathrm{m}^{+}$due to the metastable decay is completely corrected by the reflection set up. Taken from ref. 18 . 

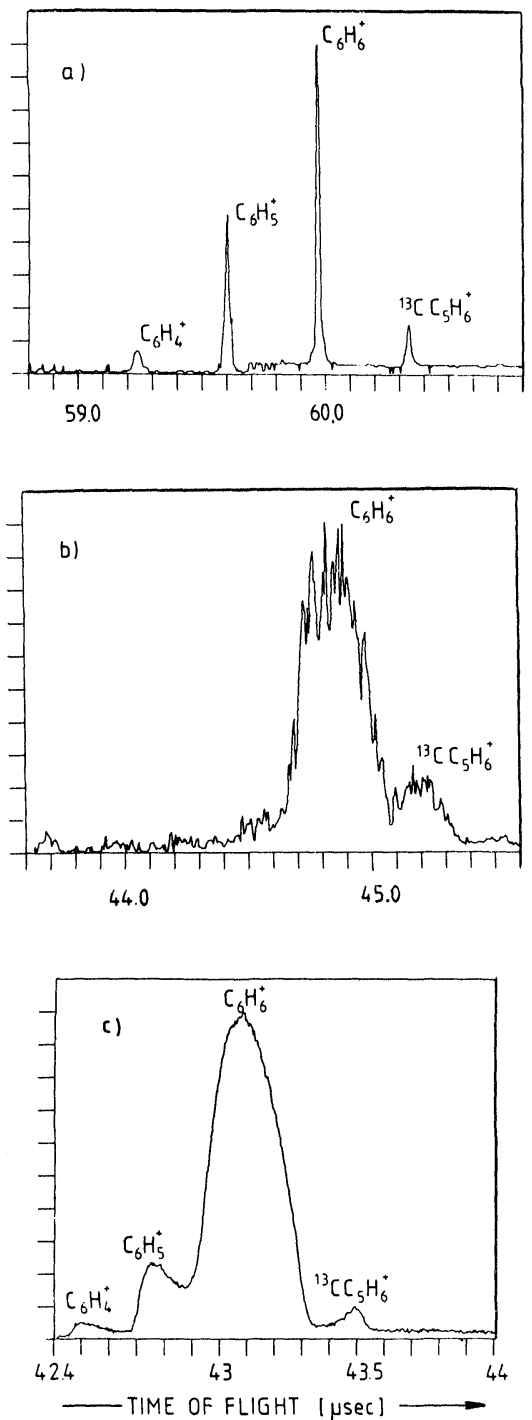

FIGURE 5 $(\mathrm{a}, \mathrm{b})$ Part of the multi-photon time-of-flight mass spectrum of benzene at $2590.1 \mathrm{~A}$ as obtained (a) with the energy correcting time-of-flight instrument, (b) with the same instrument operated in the conventional mode without energy correction (hard reflection). Note the improvement of mass resolution with the soft reflecting field and the absence of the signals of $\mathrm{C}_{6} \mathrm{H}_{5}^{+}$and $\mathrm{C}_{6} \mathrm{H}_{4}^{+}$in the uncorrected spectrum. (c) Part of the uncorrected MPI spectrum of benzene at $2529.8 \mathrm{~A}$. Note the asymmetrically broadened mass peaks $\mathrm{C}_{6} \mathrm{H}_{5}^{+}$and $\mathrm{C}_{6} \mathrm{H}_{4}^{+}$. 

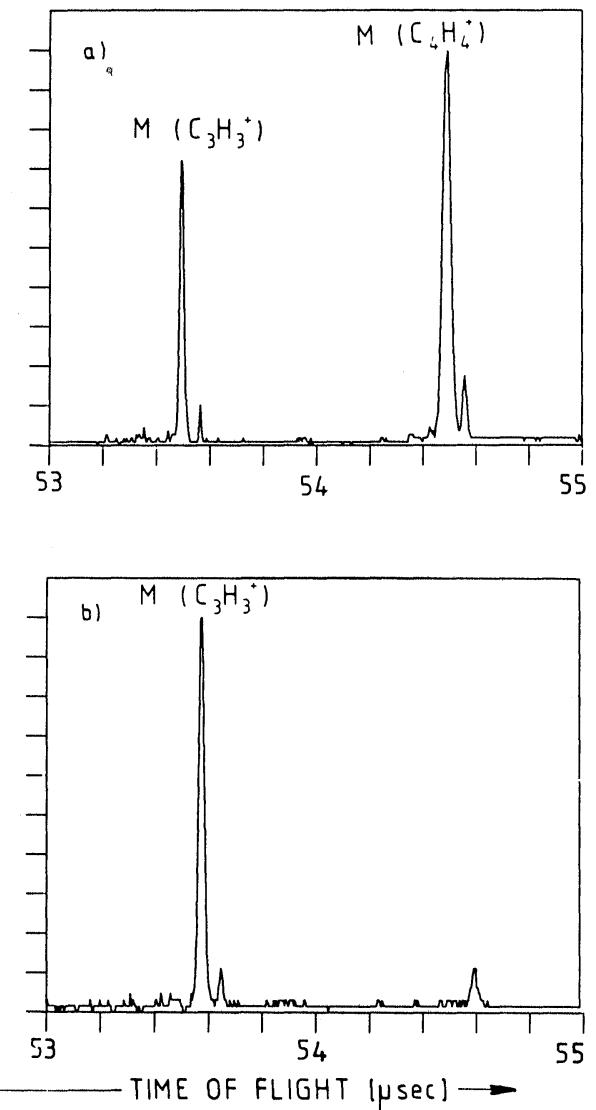

FIGURE 6 Part of the MPI mass spectrum of $\mathrm{C}_{6} \mathrm{H}_{6}$ for two different reflecting voltages. (a) For $U_{\text {refl }}=0.67 \times U_{\text {acc }}$ the $\mathrm{C}_{3} \mathrm{H}_{3}^{+}$and $\mathrm{C}_{4} \mathrm{H}_{4}^{+}$fragment ions which have been produced by a metastable decay in the drift region are observed. (b) For $U_{\text {reff }}=0.655 \times U_{\text {acc }}$ the $\mathrm{C}_{4} \mathrm{H}_{4}^{+}$ion peak has disappeared due to the somewhat larger kinetic energy of these ions with respect to the reflecting potential.

corresponding part of the uncorrected MPI time-of-flight mass spectrum of benzene for a shorter wavelength of $2529.8 \mathrm{~A}(h \nu=4.9 \mathrm{eV})$ is shown in Figure 5c for comparison. Here, two asymmetrically broadened mass peaks $\mathrm{C}_{6} \mathrm{H}_{5}^{+}$and $\mathrm{C}_{6} \mathrm{H}_{4}^{+}$are observed well separated from the benzene ions $\mathrm{C}_{6} \mathrm{H}_{6}^{+}$. From this result we conclude that for this shorter wavelength the decay processes to an observable amount 
already occur in the acceleration region of the TOF mass spectrometer. (For discussion of this result see below.)

The precursor ion of a metastable decay occurring in the drift tube is determined by increasing the reflecting field until hard reflection takes place. Under these conditions which corresponds to the case of no correction (see Figure $4 \mathrm{~b}$ ) the signals of fragments and precursor ions overlap and the precursor ion mass is determined. Then the corresponding fragment mass is determined by measuring the difference of the kinetic energy of the fragment ions and their precursor ions. This can be performed with the reflecting field acting as an energy analyzer. ${ }^{16,18}$ Reducing the reflecting voltage the ions with larger kinetic energy with respect to the reflecting potential will hit the back wall of the reflector, are discharged there and disappear. This is illustrated in Figure 6 for those ions $\mathrm{C}_{4} \mathrm{H}_{4}^{+}$that have been produced by a metastable decay in the field free drift region. For a reflecting potential which is $67 \%$ of the acceleration voltage $\left(0.67 \times e U_{\text {acc }}\right)$ the ions $\mathrm{C}_{4} \mathrm{H}_{4}^{+}$(entitled " $\mathrm{M}\left(\mathrm{C}_{4} \mathrm{H}_{4}^{+}\right)$") and $\mathrm{C}_{3} \mathrm{H}_{3}^{+}$(entitled " $\mathrm{M}\left(\mathrm{C}_{3} \mathrm{H}_{3}^{+}\right)$") which have been produced in the drift region are observed in the time-of-flight spectrum whereas all ions produced in the laser focus have disappeared (Figure 6a). This is due to the smaller kinetic energy of these metastable ions with respect to $e U_{\text {acc }}(66.6 \%$ and $50 \%$, respectively, according to the mass change in the metastable dissociation process). For the smaller reflecting potential of $0.655 \times e U_{\text {acc }}$ the ions $\mathrm{M}\left(\mathrm{C}_{4} \mathrm{H}_{4}^{+}\right)$produced in the drift region completely disappear whereas the ions $\mathrm{M}\left(\mathrm{C}_{3} \mathrm{H}_{3}^{+}\right)$with a smaller kinetic energy are reflected and reach the detector. Results for other metastable fragment ions have been shown in our previous work. ${ }^{16}$ In this way the kinetic mean energy of ions can be determined with an accuracy of at least $0.5 \%{ }^{18}$

With the methods discussed above we were able to observe the metastable elimination of $\mathrm{H}$ and $\mathrm{H}_{2}$, as well as $\mathrm{C}_{2} \mathrm{H}_{2}$ and $\mathrm{C}_{3} \mathrm{H}_{3}$ from the benzene cation $\mathrm{C}_{6} \mathrm{H}_{6}^{+}$in the MPI mass spectrum of benzene at a wavelength of $2590.1 \mathrm{~A}\left(6_{0}^{1}\right.$-band of the $S_{1} \leftarrow S_{0}$ system of benzene, $h \nu=4.786 \mathrm{eV}) .{ }^{16,18}$ According to the heats of formation of the ions and molecules under consideration ${ }^{19}$ these four metastable fragmentation processes should occur from that benzene ions which have absorbed two UV photons of $4.786 \mathrm{eV}$ as neutral and then one photon after ionization. The observation of intense and sharp signals from the metastable ions $\mathrm{C}_{6} \mathrm{H}_{5}^{+}, \mathrm{C}_{6} \mathrm{H}_{4}^{+}, \mathrm{C}_{4} \mathrm{H}_{4}^{+}$and $\mathrm{C}_{3} \mathrm{H}_{3}^{+}$in the corrected spectrum, the absence of a broadened wing of the signals $\mathrm{C}_{4} \mathrm{H}_{4}^{+}$and 
$\mathrm{C}_{3} \mathrm{H}_{3}^{+}$produced in the laser focus ${ }^{18}$ as well as the absence of the signals of $\mathrm{C}_{6} \mathrm{H}_{5}^{+}$and $\mathrm{C}_{6} \mathrm{H}_{4}^{+}$in the uncorrected spectrum ${ }^{16}$ (Figure 5a, b) suggest that these metastable fragmentation processes occur for the most part in the field free drift region. From this we conclude that within the experimental error the decay behavior can be described by a single-exponential decay characteristic for particles with a rather defined internal energy rather than for a broad internal energy distribution. Therefore, this result excludes the possibility that the parent ions may be produced by ionization from an energy level far above the ionization threshold, i.e., by absorption of an additional photon above the ionization potential within the netural molecule. We estimated a decay rate constant of about $10^{5} \mathrm{~s}^{-1} \cdot{ }^{16} \mathrm{~A}$ comparison of this value with calculated RRKM rates ${ }^{13}$ and recent measurements based on the PIPECO technique ${ }^{20,21}$ as well as the charge exchange technique $^{22,23}$ shows good agreement of our estimated value and suggests that the defined internal energy of the metastable benzene ions in our experiment is about $5 \mathrm{eV}$. This sharp energy distribution is easily explained in terms of a ladder switching process (Figure 1b). At a wavelength of the ionizing laser light of $2590.1 \mathrm{~A}$ for example the maximum energy inaccuracy of the metastable benzene ions produced via ladder switching can be $0.3 \mathrm{eV}$, the difference of twice the photon energy $(9.57 \mathrm{eV})$ and the first ionization potential of benzene $(9.25 \mathrm{eV})$. On the basis of Franck Condon factors for two-photon ionization processes, ${ }^{24}$ however, a reasonable value of this energy inaccuracy should be much smaller. In the case of autoionization a very broad energy distribution would be expected after the absorption of three photons followed by autoionization yielding in turn a multiexponential decay with a smaller average rate constant, which would be in contradiction to our experimental results.

In our experiments we observed three distinct sorts of benzene ions: stables after the absorption of only two photons in total, metastables after the absorption of three photons in total and unstables rapidly dissociating during the laser pulse after the absorption of four photons in total; all three are an additional strong argument against autoionization tree models, i.e., for ladder switching.

In the following section we will discuss the influence of the laser wavelength on the decay rates of the four metastable fragmentation processes under consideration (formation of $\mathrm{C}_{6} \mathrm{H}_{5}^{+}, \mathrm{C}_{6} \mathrm{H}_{4}^{+}, \mathrm{C}_{4} \mathrm{H}_{4}^{+}$and $\mathrm{C}_{3} \mathrm{H}_{3}^{+}$). Therefore we investigated these metastables also at a shorter 
excitation wavelength of 2529.8 A $\left(6_{0}^{1} 1_{0}^{1}\right.$-band of the $S_{1} \leftarrow S_{0}$ transition of benzene, $h \nu=4.90 \mathrm{eV}$ ). Here the signals of the fragments $\mathrm{C}_{4} \mathrm{H}_{4}^{+}$and $\mathrm{C}_{3} \mathrm{H}_{3}^{+}$produced rapidly in the laser focus show a strong asymmetric broadening towards larger times-of-flight (Figure 7). This wing is caused by metastable formation of $\mathrm{C}_{4} \mathrm{H}_{4}^{+}$and $\mathrm{C}_{3} \mathrm{H}_{3}^{+}$in the acceleration region of the time-of-flight mass spectrometer (see Figure
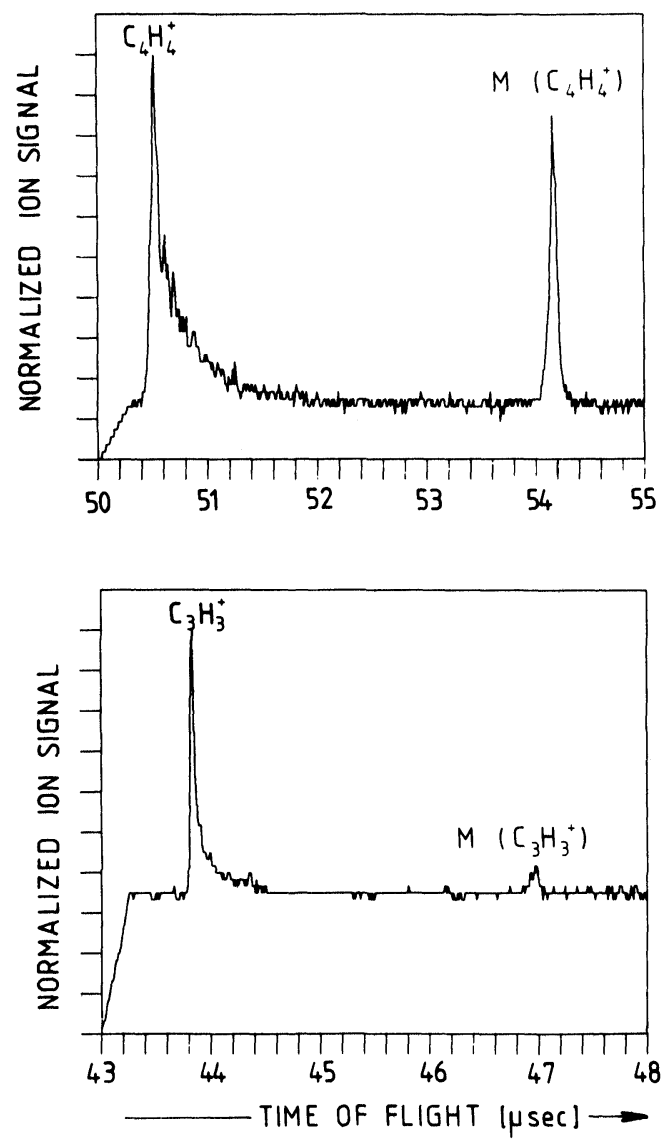

FIGURE 7 Part of the MPI mass spectrum of benzene for an excitation wavelength of $2529.8 \mathrm{~A}$ : the peak of the original fragments $\mathrm{C}_{4} \mathrm{H}_{4}^{+}$(upper trace) and $\mathrm{C}_{3} \mathrm{H}_{3}^{+}$(lower trace) show asymmetric wings due to fragments produced in the acceleration region. The ions $\mathrm{C}_{4} \mathrm{H}_{4}^{+}$and $\mathrm{C}_{3} \mathrm{H}_{3}^{+}$produced in the drift region are entitled " $\mathrm{M}\left(\mathrm{C}_{4} \mathrm{H}_{4}^{+}\right)$" and " $\mathrm{M}\left(\mathrm{C}_{3} \mathrm{H}_{3}^{+}\right)$", respectively. 
4); as demonstrated above (Figure 5c) at this shorter wavelength the metastable formation of $\mathrm{C}_{6} \mathrm{H}_{5}^{+}$and $\mathrm{C}_{6} \mathrm{H}_{4}^{+}$occurs also to an observable amount in the acceleration region of the TOF mass spectrometer. This increase of the decay rate for all four processes is readily explained by the larger photon energy with respect to the experiment at $2590.1 \mathrm{~A}$ (see above), i.e., larger internal ion energy of the metastable benzene ions $\mathrm{C}_{6} \mathrm{H}_{6}^{+}$produced via ladder switching by the absorption of three photons in total. In our laboratory detailed determination of metastable decay rate constants as function of photon energy is in progress employing the energy correcting characteristics of the reflectron TOF spectrometer. ${ }^{25}$

\section{SUMMARY AND CONCLUSION}

In summary it has been shown that the molecular parent ions produced by MPI have a well defined internal energy. This is explained in terms of a ladder switching mechanism. As a consequence thresholds for bond breaking processes and metastable decay rate constants as function of internal energy of the ions can be determined if an energy correcting reflecting field time-of-flight mass spectrometer is employed. In addition to producing a very high mass resolution the energy correcting reflectron set up greatly extends the time scale of metastable processes to be observed by the time-of-flight technique.

In conclusion multi-photon ionization is a new interesting tool in mass spectrometry with very high ionization efficiency in the laser focus. The place of ionization can be defined by the laser focus to about $10^{-5} \mathrm{~cm}^{3}$ as well as the time of ionization by the time of the laser pulse, i.e., down to typically $5 \mathrm{~ns}$. Hence, the progress of rapid chemical reactions can be monitored with good time and space resolution. The ionization conditions of space and time definitions of MPI constitute an ideal ionization source for a time-of-flight mass spectrometer. Here the time resolution of a TOF system is not degraded, as usual, by the poorly defined input conditions. In contrast to scanning instruments, where ions of only one particular mass $\mathrm{m}^{+}$and in electrostatic double focusing instruments one kinetic energy are tuned in by the deflecting fields, all ions $\mathrm{m}^{+}$with arbitrary kinetic energy are analyzed from each laser shot. This simultaneous measurement is similar to that observed in analytical Fourier transform instruments and increases the sensitivity by about two orders of magnitude. 


\section{References}

1. U. Boesl, H. J. Neusser and E. W. Schlag, Z. Naturforsch. A 33, 1546 (1978).

2. L. Zandee, R. B. Bernstein and D. A. Lichtin, J. Chem. Phvs. 69, 3427 (1978).

3. V. S. Antonov, I. N. Knyazev, V. S. Letokhov, V. M. Matiuk, V. G. Morshev and V. K. Potapov, Opt. Lett. 3, 37 (1978).

4. S. Rockwood, J. P. Reilly, K. Hohla and K. L. Kompa, Opt. Commun. 28, 175 (1979).

5. D. M. Lubman, R. Naaman and R. N. Zare, J. Chem. Phys., 72, 3034 (1980).

6. G. J. Fisanick, T. S. Eichelberger IV, B. A. Heath and M. B. Robin, J. Chem. Phys. 72, 5571 (1980).

7. C. D. Cooper, A. D. Williamson, J. C. Miller and R. N. Compton, J. Chem. Phys. 73, 1527 (1980).

8. R. S. Pandolfi, D. A. Gobell and M. A. El-Sayed, J. Phys. Chem. 85, 1779 (1981).

9. L. Zandee and R. B. Bernstein, J. Chem. Phys. 70, 2574 (1979).

10. U. Boesl, H. J. Neusser and E. W. Schlag, J. Am. Chem. Soc. 103, 5058 (1981).

11. U. Boesl, H. J. Neusser and E. W. Schlag, J. Chem. Phys. 72, 4327 (1980).

12. F. Rebentrost and A. Ben-Shaul, Chem. Phys. Lett. 77, 394 (1981).

13. W. Dietz, H. J. Neusser, U. Boesl, E. W. Schlag and S. H. Lin, Chem. Phys. 66, 105 (1982).

14. U. Boesl, H. J. Neusser and E. W. Schlag, Chem. Phys. Lett. 87, 1 (1982).

15. B. A. Mamyrin, V. I. Karataev, D. V. Shmikk and V. A. Zagulin, Zh. Eksp. Teor. Fiz. 64, 82 (1973).

16. U. Boesl, H. J. Neusser, R. Weinkauf and E. W. Schlag, J. Phys. Chem. 86, 4857 (1982).

17. R. Proch, D. M. Rider and R. N. Zare, Chem. Phys. Lett. 81, 430 (1981).

18. H. Kühlewind, H. J. Neusser and E. W. Schlag, Int. J. Mass Spectrom. Ion Phys. in press.

19. F. H. Field and J. L. Franklin, Electron Impact Phenomena (Academic Press, New York, London, 1970).

20. T. Baer, G. D. Willet, D. Smith and J. S. Phillips, J. Chem. Phys. 70, 4076 (1979).

21. J. H. D. Eland and H. Schulte, J. Chem. Phys. 62, 3835 (1975).

22. B. Andlauer and C. H. Ottinger, J. Chem. Phys. 55, 1471 (1971).

23. B. Andlauer and C. H. Ottinger, $Z$. Naturforsch. A 27, 293 (1972).

24. J. T. Meek, S. R. Long and J. P. Reilly, J. Phys. Chem. 86, 2809 (1982).

25. H. Kühlewind, H. J. Neusser and E. W. Schlag, to be published. 